\title{
NOTE ON TWO THREE-DIMENSIONAL MANIFOLDS WITH THE
}

\author{
SAME GROUP* \\ BY

\section{J. W. ALEXANDER}

1. Poincaré has proved that there exist 3-dimensional manifolds with identical Betti numbers and coefficients of torsion but which are nevertheless distinguishable in the sense of Analysis Situs by the fact that they have different groups. $\dagger$ It is proposed to set up an example of two 3-dimensional manifolds which are by no means equivalent but which cannot even be differentiated by their groups. Since the manifolds have the same group, they also have the same Betti numbers and coefficients of torsion, for in the 2-sided 3-dimensional case, these other invariants are functions of the group alone.

2. To clarify certain points that will arise later on in the discussion, we shall begin by recalling explicitly several well-known and quite obvious properties of an anchor ring, $A$, lying in ordinary 3-dimensional space.

(a) The ring $A$ may be made simply connected by means of two cuts, one along a generating circle, the other along a circle which intersects the first in one and only one point. If, therefore, we denote by $a$ and $b$ respectively the operations of describing these two curves in preassigned senses, the group of the surface will be the group generated by the operations $a$ and $b$ connected by the single relation $a b a^{-1} b^{-1} \equiv 1$. In the discussion that follows, the two circles determining $a$ and $b$ will also be referred to by the symbols for the corresponding group operations, when this can be done without confusion.

(b) The circle $a$ bounds a 2-cell that reduces the interior of the anchor ring to a simply connected piece. Any other simple closed curve with this property may be deformed continuously on the surface of the ring into coincidence with $a$.†

(c) A surface which in its initial position coincide:s with the anchor ring $A$

* Presented to the Society, September 4, 1919.

† Rendiconti di Palermo, vol. 18 (1904), p. 45. See also Dehn, Mathematische Annalen, vol. 69 (1910).

$\ddagger$ Every curve on the anchor ring which bounds in the interior region corresponds to a group operation $a^{k}$, and if the curve is to be a simple curve, we must have $k= \pm 1$, cf. Poincaré, loc. cit., pp. 26 et seq. 
may be deformed continuously within the ring until it reduces to an arbitrarily thin tube bounding as small a neighborhood as we please of some suitably chosen closed curve interior to the ring.

3. With these facts in mind, let us consider a manifold, $M$, made up of two 3-dimensional pieces separated by a common surface of genus 1 and such that either of the pieces may be mapped with its boundary on the interior and surface of an anchor ring $A$ in ordinary 3 -space. We cannot expect to crowd the entire manifold into the 3 -space without cutting it up or forcing it to overlap itself, but we can represent it conveniently in schematic form by a diagram made by mapping one of the regions in the manner indicated above and tracing on the surface $A$ of the ring the boundary $l$ of a 2-cell that reduces the omitted region to a simply connected piece.* The curve $l$, which is unique except for continuous deformations of itself $(\$ 2 b)$, determines without ambiguity the way in which the missing elements must be attached to the diagram in order to completely reconstruct the manifold. Starting with any non-bounding curve $l$ on the anchor ring, we may at once build up a manifold of the type $M$.

The invariants of the manifold can at once be read from the diagram. Let the operation of describing the curve $l$ in a properly chosen sense be $a^{\sigma} b^{r}$ $(\tau \geqq 0$ ) in terms of the fundamental operations $a$ and $b$, defined in Section $2 a$. The group of the manifold is then the group of the surface $A$ modified by the addition of the two identical relations $a \equiv 1$, and $a^{\sigma} b^{\tau} \equiv 1$, corresponding to the boundaries of the cells that reduce the two regions of the manifold to simply connected pieces. In other words, leaving out the case $\tau=0$ with which we shall not be concerned, it is the group generated by a single operation $b$ such that $b^{\tau} \equiv 1$. The Betti numbers (as defined by Poincaré) are $P_{1}=P_{2}=1$, since every curve bounds when counted $\tau$ times, and there is one coefficient of torsion, $\tau$, whenever $\tau$ is greater than one.

4. From now on, the discussion will be restricted to two particular diagrams, obtainable as follows:

Trace on the anchor ring $A$ five non-intersecting circles, each equivalent to the circle $b$ and so spaced that their points of intersection with the circle $a$ divide the latter into five equal arcs. Then, cut the anchor ring along the circle $a$, and make a continuous deformation of the cut surface into itself such that the edge of the cut containing the initial points of the $b$ curves remains fixed, while the edge containing the terminal points rotates through an angle $\frac{2}{5} \pi$. Lastly, re-join the two edges of the cut. The diagram $D$ thus obtained consists of the anchor ring $A$ and a curve $l=a b^{5}$.

The second diagram $D^{\prime}$ will be obtained in a similar way, the sole difference

* This is a simple example of Heegaard's diagrams. Cf. Heegaard, Inaugural dissertation, Copenhagen, 1898, or the French translation of the same, Bulletin de la Société Mathématique de France, vol. 44 (1916), p. 161. 
being that the edge of the cut will be rotated through the angle $\frac{4}{5} \pi$, so that the curve finally obtained will be $l^{\prime}=a^{2} b^{5}$. It may be well to observe that all the diagrams obtained in the above manner except that the edge of the cut is rotated through an angle $\left(2 \pi k+\frac{2}{5} \pi\right)$, where $k$ is any integer or zero, represent the same manifold as $D$, since any one of these diagrams (including the interior of the anchor ring) can be mapped uniformly on $D$. In a like manner, the diagrams obtained by a rotation through an angle $\left(2 \pi k+\frac{4}{5} \pi\right)$ all represent the same manifold as $D^{\prime}$. There is, however, the following essential difference between diagrams of the two classes $D$ and $D^{\prime}$ which we shall use later. In diagrams of the class $D$, the points of intersection of the two curves $a$ and $l$ are passed in the same cyclic order (12345), whether we describe one or other of the curves in the positive sense. In diagrams of the class $D^{\prime}$, if the points are passed in the order (12345) as we describe the curve $a$, they are passed in the order (13524) as we describe the curve $l^{\prime}$.

5. Let $M$ be the manifold determined by the diagram $D$. Then as an immediate consequence of Section $2 c$, we can suppose that the points of the diagram correspond to those of the entire manifold excepting the points of an arbitrarily thin tube enclosing some curve $c$ of the omitted region. Furthermore, if $\Delta$ be any other diagram defining the same manifold, we can assume, by again applying Section $2 c$, that $\Delta$ represents only that portion of the manifold within an arbitrarily thin tube about some curve $d$ which can always be so chosen as not to intersect the curve $c$. Therefore, we can suppose that the surfaces of the two diagrams correspond to non-intersecting surfaces of the manifold, and we can map the diagram $\Delta$ on the interior of the diagram $D$, in the form of a thin tube $T$ winding about in the portion of 3-space interior to the anchor ring $A$ bounding the diagram $D$.

We are now ready to show that no diagram $\Delta$ defining the manifold $M$ can possibly be the diagram $D^{\prime}$.

6. We observe that the first Betti number of the portion of 3-space occupied by the diagram $D$ and bounded by the anchor ring $A$ is 2 , since all curves in this region are homologous to zero or to the non-bounding curve $b$. Suppose we remove from this region the interior of the tube $T$. Then, however the tube $T$ may wind about, the first Betti number of the remaining region $R$ is 3 , and to each curve in the region, there can be associated a symbol

$$
\kappa a^{\prime}+\lambda b,
$$

where $a^{\prime}$ corresponds to the curve on the tube $T$ that bounds a 2-cell making the interior of the tube simply connected. A necessary condition that a curve of the region $R$ bound a 2-cell is that

$$
\kappa a^{\prime}+\lambda b \sim 0
$$


in the notation of Poincaré, or, if we use an algebraic notation in which $\boldsymbol{a}^{\prime}$ and $b$ are merely treated as linearly independent marks,

$$
\kappa a^{\prime}+\lambda b \equiv 0 \text {. }
$$

Now, the symbol for the curve $l=a b^{5}$ on the diagram $D$ is

$$
\theta a^{\prime}+5 b
$$

where $|\theta|$ denotes the number of times (algebraically), that the tube $T$ winds around the anchor ring $A$, and where the sign of $\theta$ depends on the determination of the positive direction on the curve $a^{\prime}$. Therefore, a necessary condition that a curve of the region $R$ bound a 2-cell in the entire part of the manifold $M$ exterior to the tube $T$ is that its symbol satisfy a relation

$$
\kappa a^{\prime}+\lambda b+\alpha\left(\theta a^{\prime}+5 b\right) \sim 0
$$

or, in the other notation,

$$
\kappa a^{\prime}+\lambda b \equiv 0 \bmod \left(\theta a^{\prime}+5 b\right) .
$$

Suppose $\Delta$ were the diagram $D^{\prime}$ of Section 4 . Then the curve $l^{\prime}$ of the diagram would have the symbol

$$
(5 k+2) a^{\prime} \pm 5 \theta b .
$$

For, on describing the curve $l^{\prime}$ in the positive sense, we would pass over the points where it met the curve $a^{\prime}$ in the cyclic order (13524), so that each of the five arcs of $l^{\prime}$ would wind $k+\frac{2}{5}$ times around the tube T. Moreover, since the curve $l^{\prime}$ would bound in the part of the manifold exterior to the tube $T$, we would be led, by (1), to the congruence

$$
(5 k+2) a^{\prime} \pm 5 \theta b \equiv 0 \bmod \left(\theta a^{\prime}+5 b\right) .
$$

However, it is easy to see that the congruence (2) is false. For, if $\theta=0$, we are led to $5 k+2=0$, which is false, while if $\theta \neq 0$, we are led to $\theta^{2}= \pm(5 k+2)$ which implies

$$
\theta^{2} \equiv \pm 2 \bmod 5
$$

whereas $\theta^{2}$ is always congruent to 0 or \pm 1 , modulo 5 .

Therefore, the diagram $D^{\prime}$ does not determine the same manifold as the diagram $D$.

PARIS,

December, 1918 